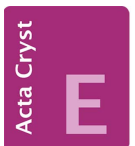

CRYSTALLOGRAPHIC COMMUNICATIONS

ISSN 2056-9890

Received 10 December 2015

Accepted 30 December 2015

Edited by M. Weil, Vienna University of Technology, Austria

Keywords: crystal structure; intermetallic compound; linear platinum chains

CCDC reference: 1444811

Supporting information: this article has supporting information at journals.iucr.org/e

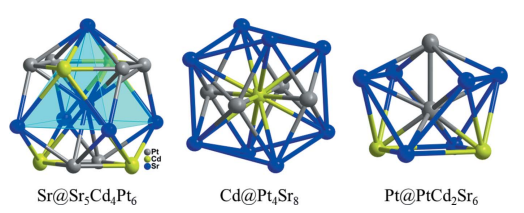

OPEN $\odot$ ACCESS

\section{Crystal structure of $\mathrm{Sr}_{2} \mathrm{CdPt}_{2}$ containing linear platinum chains}

\author{
Effendi Nawawi, ${ }^{a}$ Fakhili Gulo ${ }^{\mathrm{a} *}$ and Jürgen Köhler ${ }^{\mathrm{b}}$ \\ a Department of Chemical Education, Sriwijaya University, Inderalaya, Ogan Ilir 30662, South Sumatra, Indonesia, and \\ ${ }^{\mathbf{b}}$ Max Planck Institut für Festkörperforschung, Heisenbergstr. 1, 70698 Stuttgart, Germany. *Correspondence e-mail: \\ fgulo@unsri.ac.id
}

The ternary intermetallic title phase, distrontium cadmium diplatinum, was prepared from stoichiometric amounts of the elements at $1123 \mathrm{~K}$ for one day. The crystal structure adopts the orthorhombic $\mathrm{Ca}_{2} \mathrm{GaCu}_{2}$ structure type in space group Immm. Its main features are characterized by linear $(\mathrm{Pt}-\mathrm{Pt} \cdots \mathrm{Pt}-\mathrm{Pt})_{n}$ chains that are aligned along [010] and condensed through cadmium atoms forming Cd-centred $\mathrm{Pt}_{2} \mathrm{Cd}_{2 / 2}$ rectangles to build up sheets parallel to (001). These sheets are connected to each other via alternating (001) sheets of strontium atoms along [001]. The strontium sheets consists of corrugated $\mathrm{Sr}_{4}$ units that are condensed to each other through edge-sharing parallel to [100].

\section{Chemical context}

A large number of transition metal-based ternary intermetallic phases have been studied in terms of metal-metal interactions and structure-property relationships (Corbett, 2010). Exploratory synthetic approaches in systems $A / \mathrm{Cd} / \mathrm{Pt}$ ( $A=$ alkaline earth metal $)$ have revealed a great compositional and structural diversity. The calcium phase $\mathrm{Ca}_{6} \mathrm{Cd}_{16} \mathrm{Pt}_{8}$ contains a three-dimensional array of isolated $\mathrm{Cd}_{8}$ tetrahedral stars (TS) and a face-centred cube of $\mathrm{Pt} @ \mathrm{Ca}_{6}$ octahedra (Samal et al., 2013) whilst the structure of $\mathrm{Ca}_{6} \mathrm{Cd}_{11} \mathrm{Pt}$ crystallizes in its own structure type consisting of apically interbonded $\mathrm{Cd}_{7}$ pentagonal bipyramids and five-membered rings of $\mathrm{Ca}$ atoms (Gulo et al., 2013). The strontium phase $\mathrm{SrCd}_{4} \mathrm{Pt}_{2}$ is made up of chains of edge-sharing $\mathrm{Cd}_{4}$ tetrahedra bridged by four-bonded Sr atoms (Samal et al., 2013) and SrCdPt presents six-membered rings of $\mathrm{Sr}$ atoms in a chair conformation (Gulo \& Köhler, 2014). The barium phase $\mathrm{BaCd}_{2} \mathrm{Pt}$ exhibits zigzag chains of $\mathrm{Ba}$ atoms and Pt-centred boat and anti-boat conformations formed by six-membered rings of $\mathrm{Cd}$ atoms (Gulo \& Köhler, 2015). The Pt-based ternary intermetallic compounds with general formula $A_{2} X \mathrm{Pt}_{2}(A=$ alkaline-earth or rare-earth metal; $X=$ diel, triel, or tetrel element) adopt five different structure types. $\mathrm{Sr}_{2} \mathrm{InPt}_{2}$ (Muts et al., 2007) crystallizes in the monoclinic $\mathrm{Ca}_{2} \mathrm{Ir}_{2} \mathrm{~S}$ type (Schoolaert \& Jung, 2002), $\mathrm{Pu}_{2} \mathrm{SnPt}_{2}$ (Pereira et al., 1997) in the tetragonal $\mathrm{Mo}_{2} \mathrm{FeB}_{2}$ type (Gladyshevskii et al., 1996) while $\mathrm{U}_{2} \mathrm{CdPt}_{2}$ has its own structure type (Gravereau et al., 1994). $\mathrm{Ce}_{2} \mathrm{CdPt}_{2}$ (Pöttgen et al., 2000) adopts the tetragonal $\mathrm{U}_{3} \mathrm{Si}_{2}$ type (Zachariasen, 1948), and $\mathrm{Ca}_{2} \mathrm{CdPt}_{2}$ (Samal \& Corbett, 2012) the orthorhombic $\mathrm{Ca}_{2} \mathrm{GaCu}_{2}$ type (Fornasini \& Merlo, 1988).

In this article we present the crystal structure of the novel intermetallic phase $\mathrm{Sr}_{2} \mathrm{CdPt}_{2}$ containing linear $(\mathrm{Pt}-\mathrm{Pt} \cdots \mathrm{Pt}-$ $\mathrm{Pt})_{n}$ chains as a principal structural motif. 


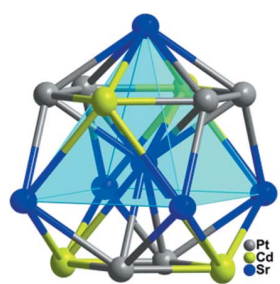

$\mathrm{Sr} @ \mathrm{Sr}_{5} \mathrm{Cd}_{4} \mathrm{Pt}_{6}$

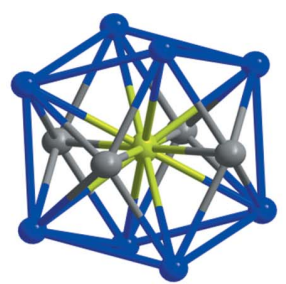

$\mathrm{Cd} @ \mathrm{Pt}_{4} \mathrm{Sr}_{8}$

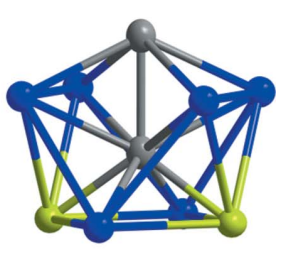

$\mathrm{Pt} @ \mathrm{PtCd}_{2} \mathrm{Sr}_{6}$
Figure 1

Coordination of strontium, cadmium, and platinum atoms in $\mathrm{Sr}_{2} \mathrm{CdPt}_{2}$. Displacement ellipsoids are displayed at the $90 \%$ probability level.

\section{Structural commentary}

The ternary intermetallic title phase adopts the orthorhombic $\mathrm{Ca}_{2} \mathrm{GaCu}_{2}$ structure type (Fornasini \& Merlo, 1988) with the $\mathrm{Ca}, \mathrm{Ga}$, and $\mathrm{Cu}$ sites replaced by $\mathrm{Sr}, \mathrm{Cd}$, and $\mathrm{Pt}$ sites, respectively. The three atoms occupy three independent sites in the unit cell. The $\mathrm{Sr}$ atom resides on a special position with site symmetry $m m 2$ (Wyckoff site $4 j$ ), the Cd atom occupies a special positions with site symmetry $\mathrm{mmm}(2 a)$ and the $\mathrm{Pt}$ atom is on a special positions with site symmetry $m 2 m(4 h)$. In the two structures, the transition metals (platinum and copper, respectively) occupy the same positions. In contrast, in the structure of $\mathrm{SrCd}_{4} \mathrm{Pt}_{2}$ (Samal et al., 2013) which is isotypic with $\mathrm{ZrFe}_{4} \mathrm{Si}_{2}$ (Yarmolyuk et al., 1975), the transition metals (platinum and iron, respectively) occupy different positions and the $\mathrm{Pt}$ atoms reside on the respective $\mathrm{Si}$ sites because silicon and platinum atoms are the most electronegative atoms in the two systems. The new phase $\mathrm{Sr}_{2} \mathrm{CdPt}_{2}$ contains 26 valence electrons and, as already mentioned, is isotypic with

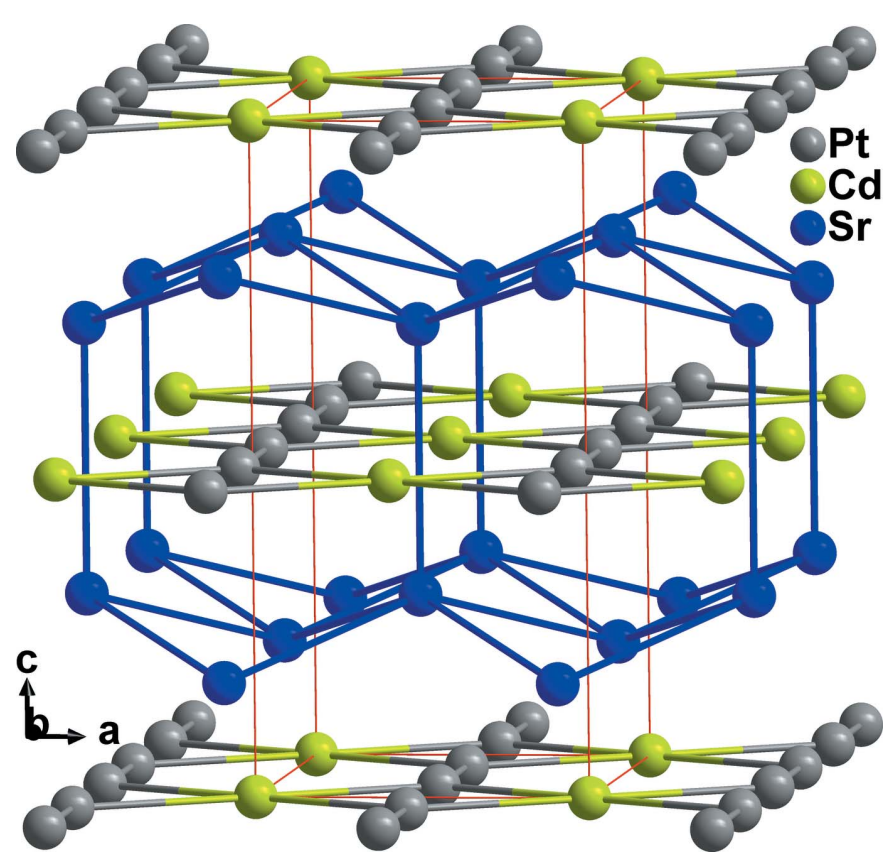

Figure 2

Projection of the crystal structure of $\mathrm{Sr}_{2} \mathrm{CdPt}_{2}$ approximately along the $b$ axis.
$\mathrm{Ca}_{2} \mathrm{GaCu}_{2}$. However, in comparison the structure of $\mathrm{Sr}_{2} \mathrm{CdPt}_{2}$ is appreciably distorted along the platinum chains, presumably because $\mathrm{Ca}_{2} \mathrm{GaCu}_{2}$ contains much smaller $\mathrm{Cu}$ atoms and a larger valence electron count of 29. Coordination spheres of each atomic site in the title structure are illustrated in Fig. 1. The $\mathrm{Sr}$ atom is coordinated by five other $\mathrm{Sr}$, four $\mathrm{Cd}$, and four $\mathrm{Pt}$ atoms. The $\mathrm{Sr}-\mathrm{Sr}$ bond lengths vary from 3.674 (3) to 3.854 (1) $\AA$, the $\mathrm{Sr}-\mathrm{Cd}$ distances range from 3.490 (1) to 3.577 (1) $\AA$, whereas the $\mathrm{Sr}-\mathrm{Pt}$ values vary only slightly, from 3.188 (1) to 3.231 (1) $\AA$. Six Sr atoms construct a squareplanar pyramid, $\mathrm{Sr}_{\mathrm{Sr}_{5}}$. The existence of $\mathrm{Sr}-\mathrm{Sr}$ strong bonds is observable in SrCdPt (Gulo \& Köhler, 2014) but not in $\mathrm{SrCd}_{4} \mathrm{Pt}_{2}$ (Samal et al., 2013). The Cd atom exhibits a coordination number of twelve and has eight $\mathrm{Sr}$ and four $\mathrm{Pt}$ atoms in its environment with $\mathrm{Cd}-\mathrm{Pt}$ distances of 2.785 (1) $\AA$. The $\mathrm{Pt}$ atom is surrounded by six $\mathrm{Sr}$, two $\mathrm{Cd}$ and one $\mathrm{Pt}$ atoms with a Pt-Pt distance of 2.734 (1) $\AA$. This distance is slightly longer than those found in $\mathrm{Ca}_{2} \mathrm{CdPt}_{2}(2.659 \AA$; Samal \& Corbett, 2012) or $\mathrm{Sr}_{2} \mathrm{InPt}_{2}$ (2.707 $\AA$; Muts et al., 2007) but shorter than those in $\mathrm{Pu}_{2} \mathrm{SnPt}_{2}$ (Pereira et al., 1997), $\mathrm{U}_{2} \mathrm{CdPt}_{2}$ (Gravereau et al., 1994) or $\mathrm{Ce}_{2} \mathrm{CdPt}_{2}$ (Pöttgen et al., 2000). All other interatomic distances $(\mathrm{Sr}-\mathrm{Cd}, \mathrm{Sr}-\mathrm{Pt}$, and $\mathrm{Cd}-\mathrm{Pt})$ are in agreement with those found in some ternary compounds in $A / \mathrm{Cd} / \mathrm{Pt}$ systems $(A=$ alkaline earth metal $)$.

\section{Packing features}

$\mathrm{Sr}$ atoms are bound together into corrugated sheets consisting of edge-sharing $\mathrm{Sr}_{4}$-units. These sheets spread parallel to (001) and are linked by another $\mathrm{Sr}-\mathrm{Sr}$ bond of 3.674 (3) $\AA$ along [001]. (Fig. 2). The crystal structure is also characterized by the existence of linear $(\mathrm{Pt}-\mathrm{Pt} \cdots \mathrm{Pt}-\mathrm{Pt})_{n}$ chains along [010] with longer distances of 3.2010 (14) $\AA$ between pairs of tightly

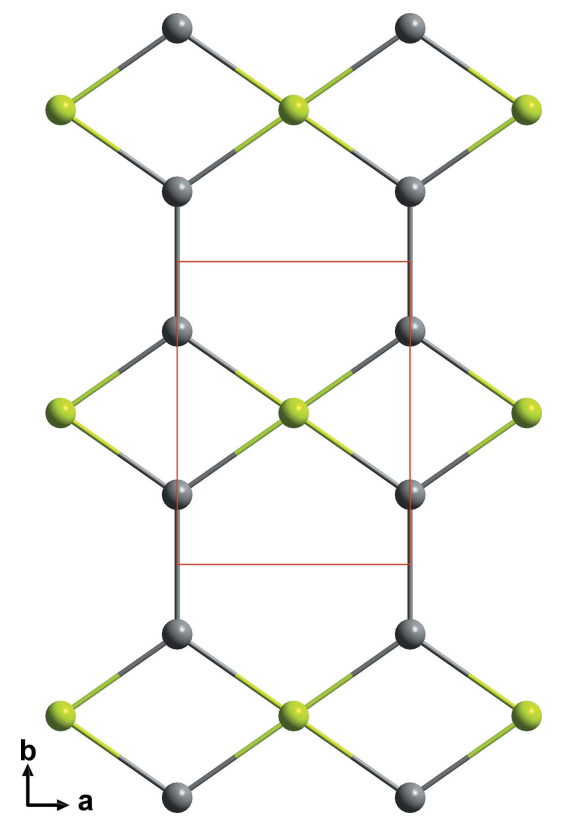

Figure 3

Projection of linear platinum chains that are aligned along the $b$ axis and condensed via cadmium atoms forming $\mathrm{Pt}_{2} \mathrm{Cd}_{2 / 2}$-rectangles in the $a b$ plane 
Table 1

Experimental details.

\begin{tabular}{|c|c|}
\hline \multicolumn{2}{|l|}{ Crystal data } \\
\hline Chemical formula & $\mathrm{CdPt}_{2} \mathrm{Sr}_{2}$ \\
\hline$M_{\mathrm{r}}$ & 677.82 \\
\hline Crystal system, space group & Orthorhombic, Immm \\
\hline Temperature $(\mathrm{K})$ & 293 \\
\hline$a, b, c(\AA)$ & $4.5596(9), 5.9351(12), 9.1874(18)$ \\
\hline$V\left(\AA^{3}\right)$ & $248.63(9)$ \\
\hline$Z$ & 2 \\
\hline Radiation type & Мо $K \alpha$ \\
\hline$\mu\left(\mathrm{mm}^{-1}\right)$ & 81.39 \\
\hline Crystal size $(\mathrm{mm})$ & $0.08 \times 0.06 \times 0.05$ \\
\hline \multicolumn{2}{|l|}{ Data collection } \\
\hline Diffractometer & Bruker P4 \\
\hline Absorption correction & $\begin{array}{l}\text { Multi-scan (SADABS; Bruker, } \\
\text { 2001) }\end{array}$ \\
\hline$T_{\min }, T_{\max }$ & $0.004,0.017$ \\
\hline $\begin{array}{l}\text { No. of measured, independent and } \\
\text { observed }[I>2 \sigma(I)] \text { reflections }\end{array}$ & $1069,190,172$ \\
\hline$R_{\text {int }}$ & 0.025 \\
\hline$(\sin \theta / \lambda)_{\max }\left(\AA^{-1}\right)$ & 0.666 \\
\hline \multicolumn{2}{|l|}{ Refinement } \\
\hline$R\left[F^{2}>2 \sigma\left(F^{2}\right)\right], w R\left(F^{2}\right), S$ & $0.022,0.058,1.16$ \\
\hline No. of reflections & 190 \\
\hline No. of parameters & 13 \\
\hline$\Delta \rho_{\max }, \Delta \rho_{\min }\left(\mathrm{e} \AA^{-3}\right)$ & $1.84,-2.51$ \\
\hline
\end{tabular}

Computer programs: XSCANS (Bruker, 2001), SHELXS97 and SHELXL97 (Sheldrick, 2008) and DIAMOND (Brandenburg, 2010).

bound $\mathrm{Pt}-\mathrm{Pt}$ dumbbells, a significant distortion in the direction of dimerization. The platinum chains are condensed into (001) sheets through $\mathrm{Cd}$ atoms, forming Cd-centred rectangles with composition $\mathrm{Pt}_{2} \mathrm{Cd}_{2 / 2}$ (Fig. 3). The $\mathrm{Pt}_{2} \mathrm{Cd}_{2 / 2}$ layers are stacked along [001] and are linked through the corrugated sheets of Sr atoms.

\section{Database survey}

A search of the Pearson's Crystal Data - Crystal Structure Database for Inorganic Compounds (Villars \& Cenzual, 2015) for the $\mathrm{Sr} / \mathrm{Cd} / \mathrm{Pt}$ family of compounds returned two compounds only: $\mathrm{SrCd}_{4} \mathrm{Pt}_{2}$ (Samal et al., 2013) and $\mathrm{SrCdPt}$ (Gulo \& Köhler, 2014).

\section{Synthesis and crystallization}

The title compound was synthesized from starting materials of Sr granules (99.9+\%, Alfa Aesar), Cd powder (99.9+\%, Alfa Aesar) and Pt powder (99.95\%, Chempur). A stoichiometric mixture of these elements was loaded into a $\mathrm{Nb}$ ampoule in an Ar-filled dry box. The $\mathrm{Nb}$ ampoule was then weld-sealed under an Ar atmosphere and subsequently enclosed in an evacuated silica jacket. The sample was then heated to $1123 \mathrm{~K}$ for $15 \mathrm{~h}$, equilibrated at $923 \mathrm{~K}$ for 4 days, and followed by slow cooling to room temperature.

\section{Refinement}

Crystal data, data collection and structure refinement details are summarized in Table 1 . The maximum and minimum remaining electron densities are located 1.66 and $0.81 \AA$, respectively, from the Pt site.

\section{Acknowledgements}

Financial support for EN and FG from PNBP Unsri is gratefully acknowledged.

\section{References}

Brandenburg, K. (2010). DIAMOND. Crystal Impact GbR, Bonn, Germany.

Bruker (2001). XSCANS and $S A D A B S$. Bruker AXS Inc., Madison, Wisconsin, USA.

Corbett, J. D. (2010). Inorg. Chem. 49, 13-28.

Fornasini, M. L. \& Merlo, F. (1988). J. Less-Common Met. 142, 289 294.

Gladyshevskii, E. I., Fedorov, T. F., Kuz'ma, Y. B. \& Skolozdra, R. V. (1996). Sov. Powder Metall. Met. Ceram. 5, 305-309.

Gravereau, P., Mirambet, F., Chevalier, B., Weill, F., Fournès, L., Laffargue, D., Bourée, F. \& Etourneau, J. R. (1994). J. Mater. Chem. 4, 1893-1895.

Gulo, F. \& Köhler, J. (2014). Acta Cryst. E70, 590-592.

Gulo, F. \& Köhler, J. (2015). Z. Anorg. Allg. Chem. 641, 557-560.

Gulo, F., Samal, S. L. \& Corbett, J. D. (2013). Inorg. Chem. 52, 1011210118.

Muts, I. R., Nilges, T., Rodewald, U. C., Zaremba, V. I. \& Pöttgen, R. (2007). Z. Naturforsch. B, 62, 1563-1566.

Pereira, L. C. J., Wastin, F., Winand, J. M., Kanellakopoulos, B., Rebizant, J., Spirlet, J. C. \& Almeida, M. (1997). J. Solid State Chem. 134, 138-147.

Pöttgen, R., Fugmann, A., Hoffmann, R. D., Rodewald, U. C. \& Niepmann, D. (2000). Z. Naturforsch. B, 55, 155-161.

Samal, S. L. \& Corbett, J. D. (2012). Z. Anorg. Allg. Chem. 638, $1963-$ 1969.

Samal, S. L., Gulo, F. \& Corbett, J. D. (2013). Inorg. Chem. 52, 26972704.

Schoolaert, S. \& Jung, W. (2002). Z. Anorg. Allg. Chem. 628, 18061810.

Sheldrick, G. M. (2008). Acta Cryst. A64, 112-122.

Villars, P. \& Cenzual, K. (2015). Pearson's Crystal Data - Crystal Structure Database for Inorganic Compounds. Release 2015/16. ASM International, Materials Park, Ohio, USA.

Yarmolyuk, Y. P., Lysenko, L. A. \& Gladyshevskii, E. I. (1975). Uopov. Akad. Nauk Ukr. RSR, Ser. A. 279.

Zachariasen, W. H. (1948). Acta Cryst. 1, 265-268. 


\section{supporting information}

Acta Cryst. (2016). E72, 144-146 [doi:10.1107/S2056989015024937]

\section{Crystal structure of $\mathrm{Sr}_{2} \mathrm{CdPt}_{2}$ containing linear platinum chains}

\section{Effendi Nawawi, Fakhili Gulo and Jürgen Köhler}

\section{Computing details}

Data collection: XSCANS (Bruker, 2001); cell refinement: XSCANS (Bruker, 2001); data reduction: XSCANS (Bruker, 2001); program(s) used to solve structure: SHELXS97 (Sheldrick, 2008); program(s) used to refine structure: SHELXL97 (Sheldrick, 2008); molecular graphics: DIAMOND (Brandenburg, 2010); software used to prepare material for publication: SHELXL97 (Sheldrick, 2008).

Distrontium cadmium diplatinum

Crystal data

$\mathrm{CdPt}_{2} \mathrm{Sr}_{2}$

$M_{r}=677.82$

Orthorhombic, Immm

Hall symbol: -I 22

$a=4.5596(9) \AA$

$b=5.9351$ (12) $\AA$

$c=9.1874(18) \AA$

$V=248.63(9) \AA^{3}$

$Z=2$

Data collection

Bruker P4 4-circle diffractometer

Radiation source: fine-focus sealed tube

Graphite monochromator

Detector resolution: 0 pixels $\mathrm{mm}^{-1}$

$\omega$-scans

Absorption correction: multi-scan (SADABS; Bruker, 2001)

$T_{\min }=0.004, T_{\max }=0.017$

\section{Refinement}

Refinement on $F^{2}$

Least-squares matrix: full

$R\left[F^{2}>2 \sigma\left(F^{2}\right)\right]=0.022$

$w R\left(F^{2}\right)=0.058$

$S=1.16$

190 reflections

13 parameters

0 restraints

Primary atom site location: structure-invariant direct methods
$F(000)=560$

$D_{\mathrm{x}}=9.054 \mathrm{Mg} \mathrm{m}^{-3}$

Mo $K \alpha$ radiation, $\lambda=0.71073 \AA$

Cell parameters from 25 reflections

$\theta=12-18^{\circ}$

$\mu=81.39 \mathrm{~mm}^{-1}$

$T=293 \mathrm{~K}$

Block, brown

$0.08 \times 0.06 \times 0.05 \mathrm{~mm}$

1069 measured reflections

190 independent reflections

172 reflections with $I>2 \sigma(I)$

$R_{\text {int }}=0.025$

$\theta_{\max }=28.3^{\circ}, \theta_{\min }=4.1^{\circ}$

$h=-5 \rightarrow 6$

$k=-7 \rightarrow 7$

$l=-11 \rightarrow 11$

Secondary atom site location: difference Fourier map

$w=1 /\left[\sigma^{2}\left(F_{\mathrm{o}}^{2}\right)+(0.0337 P)^{2}+3.9776 P\right]$

where $P=\left(F_{\mathrm{o}}^{2}+2 F_{\mathrm{c}}^{2}\right) / 3$

$(\Delta / \sigma)_{\max }<0.001$

$\Delta \rho_{\max }=1.84$ e $\AA^{-3}$

$\Delta \rho_{\min }=-2.51$ e $\AA^{-3}$

Extinction correction: SHELXL, $\mathrm{Fc}^{*}=\mathrm{kFc}\left[1+0.001 \times \mathrm{Fc}^{2} \lambda^{3} / \sin (2 \theta)\right]^{-1 / 4}$

Extinction coefficient: 0.0024 (4) 


\section{Special details}

Geometry. All e.s.d.'s (except the e.s.d. in the dihedral angle between two l.s. planes) are estimated using the full covariance matrix. The cell e.s.d.'s are taken into account individually in the estimation of e.s.d.'s in distances, angles and torsion angles; correlations between e.s.d.'s in cell parameters are only used when they are defined by crystal symmetry. An approximate (isotropic) treatment of cell e.s.d.'s is used for estimating e.s.d.'s involving 1.s. planes.

Refinement. Refinement of $F^{2}$ against ALL reflections. The weighted $R$-factor $w R$ and goodness of fit $S$ are based on $F^{2}$, conventional $R$-factors $R$ are based on $F$, with $F$ set to zero for negative $F^{2}$. The threshold expression of $F^{2}>\sigma\left(F^{2}\right)$ is used only for calculating $R$-factors (gt) etc. and is not relevant to the choice of reflections for refinement. $R$-factors based on $F^{2}$ are statistically about twice as large as those based on $F$, and $R$ - factors based on ALL data will be even larger.

Fractional atomic coordinates and isotropic or equivalent isotropic displacement parameters $\left(\hat{A}^{2}\right)$

\begin{tabular}{lllll}
\hline & $x$ & $y$ & $z$ & $U_{\text {iso }} * U_{\text {eq }}$ \\
\hline $\mathrm{Pt}$ & 0.0000 & $0.23034(10)$ & 0.5000 & $0.0172(3)$ \\
$\mathrm{Cd}$ & -0.5000 & 0.5000 & 0.5000 & $0.0172(4)$ \\
$\mathrm{Sr}$ & 0.0000 & 0.5000 & $0.19995(16)$ & $0.0162(4)$ \\
\hline
\end{tabular}

Atomic displacement parameters $\left(\AA^{2}\right)$

\begin{tabular}{lllllll}
\hline & $U^{11}$ & $U^{22}$ & $U^{33}$ & $U^{12}$ & $U^{13}$ & $U^{23}$ \\
\hline $\mathrm{Pt}$ & $0.0156(4)$ & $0.0205(4)$ & $0.0155(4)$ & 0.000 & 0.000 & 0.000 \\
$\mathrm{Cd}$ & $0.0157(8)$ & $0.0152(8)$ & $0.0207(9)$ & 0.000 & 0.000 & 0.000 \\
$\mathrm{Sr}$ & $0.0171(8)$ & $0.0169(8)$ & $0.0146(8)$ & 0.000 & 0.000 & 0.000 \\
\hline
\end{tabular}

Geometric parameters $\left(\stackrel{A}{\circ}{ }^{\circ}\right)$

\begin{tabular}{|c|c|c|c|}
\hline $\mathrm{Pt}-\mathrm{Pt}^{\mathrm{i}}$ & $2.7341(13)$ & $\mathrm{Cd}-\mathrm{Sr}^{\mathrm{vii}}$ & $3.4901(10)$ \\
\hline $\mathrm{Pt}-\mathrm{Cd}^{\mathrm{ii}}$ & $2.7855(5)$ & $\mathrm{Cd}-\mathrm{Sr}^{\mathrm{viii}}$ & $3.5773(12)$ \\
\hline $\mathrm{Pt}-\mathrm{Cd}$ & $2.7855(5)$ & $\mathrm{Cd}-\mathrm{Sr}^{\mathrm{iii}}$ & $3.5773(13)$ \\
\hline $\mathrm{Pt}-\mathrm{Sr}^{\mathrm{iii}}$ & $3.1876(14)$ & $\mathrm{Cd}-\mathrm{Sr}$ & $3.5773(12)$ \\
\hline $\mathrm{Pt}-\mathrm{Sr}$ & $3.1876(14)$ & $\mathrm{Cd}-\mathrm{Sr}^{\mathrm{ix}}$ & $3.5773(13)$ \\
\hline $\mathrm{Pt}-\mathrm{Pt}^{\mathrm{iii}}$ & $3.2010(14)$ & $\mathrm{Sr}-\mathrm{Pt}^{\mathrm{iii}}$ & $3.1876(14)$ \\
\hline $\mathrm{Pt}-\mathrm{Sr}^{\mathrm{iv}}$ & $3.2312(10)$ & $\mathrm{Sr}-\mathrm{Pt}^{\mathrm{iv}}$ & $3.2312(10)$ \\
\hline $\mathrm{Pt}-\mathrm{Sr}^{\mathrm{v}}$ & $3.2312(10)$ & $\mathrm{Sr}-\mathrm{Pt}^{\mathrm{xii}}$ & $3.2312(10)$ \\
\hline $\mathrm{Pt}-\mathrm{Sr}^{\mathrm{vi}}$ & $3.2312(10)$ & $\mathrm{Sr}-\mathrm{Pt}^{\mathrm{xiii}}$ & $3.2312(10)$ \\
\hline $\mathrm{Pt}-\mathrm{Sr}^{\mathrm{vii}}$ & $3.2312(10)$ & $\mathrm{Sr}-\mathrm{Pt}^{\mathrm{v}}$ & $3.2312(10)$ \\
\hline $\mathrm{Cd}-\mathrm{Pt}^{\mathrm{iii}}$ & $2.7855(5)$ & $\mathrm{Sr}-\mathrm{Cd}^{\mathrm{xiv}}$ & $3.4901(10)$ \\
\hline $\mathrm{Cd}-\mathrm{Pt}^{\text {viii }}$ & $2.7855(5)$ & $\mathrm{Sr}-\mathrm{Cd}^{\mathrm{xiii}}$ & $3.4901(10)$ \\
\hline $\mathrm{Cd}-\mathrm{Pt}^{\mathrm{tix}}$ & $2.7855(5)$ & $\mathrm{Sr}-\mathrm{Cd}^{\mathrm{ii}}$ & $3.5773(12)$ \\
\hline $\mathrm{Cd}-\mathrm{Sr}^{\mathrm{iv}}$ & $3.4901(10)$ & $\mathrm{Sr}-\mathrm{Sr}^{\mathrm{Xv}}$ & $3.674(3)$ \\
\hline $\mathrm{Cd}-\mathrm{Sr}^{\mathrm{x}}$ & $3.4901(10)$ & $\mathrm{Sr}-\mathrm{Sr}^{\mathrm{xvi}}$ & $3.8535(9)$ \\
\hline $\mathrm{Cd}-\mathrm{Sr}^{\mathrm{xi}}$ & $3.4901(10)$ & & \\
\hline $\mathrm{Pt}-\mathrm{Pt}-\mathrm{Cd}^{\mathrm{ii}}$ & $125.070(13)$ & $\mathrm{Sr}^{\mathrm{viii}}-\mathrm{Cd}-\mathrm{Sr}^{\mathrm{iii}}$ & 180.0 \\
\hline $\mathrm{Pt}^{\mathrm{i}}-\mathrm{Pt}-\mathrm{Cd}$ & $125.070(13)$ & $\mathrm{Pt}^{\mathrm{iii}}-\mathrm{Cd}-\mathrm{Sr}$ & $58.560(14)$ \\
\hline $\mathrm{Cd}^{\mathrm{ii}}-\mathrm{Pt}-\mathrm{Cd}$ & $109.86(3)$ & $\mathrm{Pt}^{\mathrm{viii}}-\mathrm{Cd}-\mathrm{Sr}$ & $121.440(14)$ \\
\hline $\mathrm{Pt}-\mathrm{Pt}-\mathrm{Sr}^{\mathrm{iii}}$ & $120.139(18)$ & $\mathrm{Pt}^{\mathrm{ix}}-\mathrm{Cd}-\mathrm{Sr}$ & $121.440(14)$ \\
\hline $\mathrm{Cd}^{\mathrm{ii}}-\mathrm{Pt}-\mathrm{Sr}^{\mathrm{iii}}$ & $73.232(13)$ & $\mathrm{Pt}-\mathrm{Cd}-\mathrm{Sr}$ & $58.560(14)$ \\
\hline $\mathrm{Cd}-\mathrm{Pt}-\mathrm{Sr}^{\mathrm{iii}}$ & $73.232(13)$ & $\mathrm{Sr}^{\mathrm{iv}}-\mathrm{Cd}-\mathrm{Sr}$ & $66.071(12)$ \\
\hline
\end{tabular}




$$
\begin{aligned}
& \mathrm{Pt}^{\mathrm{i}}-\mathrm{Pt}-\mathrm{Sr} \\
& \mathrm{Cd}^{\mathrm{ii}}-\mathrm{Pt}-\mathrm{Sr} \\
& \mathrm{Cd}-\mathrm{Pt}-\mathrm{Sr} \\
& \mathrm{Sr} \text { iii- } \mathrm{Pt}-\mathrm{Sr} \\
& \mathrm{Pt}^{\mathrm{i}}-\mathrm{Pt}-\mathrm{Pt}^{\mathrm{tii}} \\
& \mathrm{Cd}^{\mathrm{ii}}-\mathrm{Pt}-\mathrm{Pt}^{\mathrm{iii}} \\
& \mathrm{Cd}-\mathrm{Pt}-\mathrm{Pt}^{\mathrm{iii}} \\
& \mathrm{Sr}^{\mathrm{iii}}-\mathrm{Pt}-\mathrm{Pt}^{\mathrm{iii}} \\
& \mathrm{Sr}-\mathrm{Pt}-\mathrm{Pt}^{\mathrm{iii}} \\
& \mathrm{Pt}^{\mathrm{i}}-\mathrm{Pt}-\mathrm{Sr}^{\mathrm{iv}} \\
& \mathrm{Cd}^{\mathrm{ii}}-\mathrm{Pt}-\mathrm{Sr}^{\mathrm{iv}} \\
& \mathrm{Cd}-\mathrm{Pt}-\mathrm{Sr}^{\mathrm{iv}} \\
& \mathrm{Sr}^{\mathrm{iii}}-\mathrm{Pt}-\mathrm{Sr}^{\mathrm{iv}} \\
& \mathrm{Sr}-\mathrm{Pt}-\mathrm{Sr}^{\text {iv }} \\
& \mathrm{Pt}^{\mathrm{iii}}-\mathrm{Pt}-\mathrm{Sr}^{\mathrm{iv}} \\
& \mathrm{Pt}^{\mathrm{i}}-\mathrm{Pt}-\mathrm{Sr}^{\mathrm{v}} \\
& \mathrm{Cd}^{\mathrm{ii}}-\mathrm{Pt}-\mathrm{Sr}^{\mathrm{v}} \\
& \mathrm{Cd}-\mathrm{Pt}-\mathrm{Sr}^{\mathrm{v}} \\
& \mathrm{Sr}^{\mathrm{iii}}-\mathrm{Pt}-\mathrm{Sr}^{\mathrm{v}} \\
& \mathrm{Sr}-\mathrm{Pt}-\mathrm{Sr}^{\mathrm{v}} \\
& \mathrm{Pt}^{\mathrm{iii}}-\mathrm{Pt}-\mathrm{Sr}^{\mathrm{v}} \\
& \mathrm{Sr}^{\text {iv }}-\mathrm{Pt}-\mathrm{Sr}^{\mathrm{v}} \\
& \mathrm{Pt}^{\mathrm{i}}-\mathrm{Pt}-\mathrm{Sr}^{\mathrm{vi}} \\
& \mathrm{Cd}^{\mathrm{ii}}-\mathrm{Pt}-\mathrm{Sr}^{\mathrm{vi}} \\
& \mathrm{Cd}-\mathrm{Pt}-\mathrm{Sr}^{\mathrm{vi}} \\
& \mathrm{Sr}^{\mathrm{iii}}-\mathrm{Pt}-\mathrm{Sr}^{\mathrm{vi}} \\
& \mathrm{Sr}-\mathrm{Pt}-\mathrm{Sr}^{\mathrm{vi}} \\
& \mathrm{Pt}^{\mathrm{iii}}-\mathrm{Pt}-\mathrm{Sr}^{\mathrm{vi}} \\
& \mathrm{Sr}^{\text {iv }}-\mathrm{Pt}-\mathrm{Sr}^{\text {vi }} \\
& \mathrm{Sr}^{\mathrm{v}}-\mathrm{Pt}-\mathrm{Sr}^{\mathrm{vi}} \\
& \mathrm{Pt}^{\mathrm{i}}-\mathrm{Pt}-\mathrm{Sr}^{\mathrm{vii}} \\
& \mathrm{Cd}^{\mathrm{di}}-\mathrm{Pt}-\mathrm{Sr}^{\mathrm{vii}} \\
& \mathrm{Cd}-\mathrm{Pt}-\mathrm{Sr}^{\mathrm{vii}} \\
& \mathrm{Sr}^{\mathrm{iii}}-\mathrm{Pt}-\mathrm{Sr}^{\mathrm{vii}} \\
& \mathrm{Sr}-\mathrm{Pt}-\mathrm{Sr}^{\mathrm{vii}} \\
& \mathrm{Pt}^{\mathrm{iii}}-\mathrm{Pt}-\mathrm{Sr}^{\mathrm{rii}} \\
& \mathrm{Sr}^{\text {iv }}-\mathrm{Pt}-\mathrm{Sr}^{\text {vii }} \\
& \mathrm{Sr}^{\mathrm{v}}-\mathrm{Pt}-\mathrm{Sr}^{\mathrm{vii}} \\
& \mathrm{Sr}^{\mathrm{vi}}-\mathrm{Pt}-\mathrm{Sr}^{\mathrm{vii}} \\
& \mathrm{P}^{\mathrm{tiii}}-\mathrm{Cd}-\mathrm{P}^{\mathrm{tiii}} \\
& \mathrm{Pt}^{\mathrm{iii}}-\mathrm{Cd}-\mathrm{Pt}^{\mathrm{ix}} \\
& \mathrm{Pt}^{\mathrm{v}}{ }^{\mathrm{iii}}-\mathrm{Cd}-\mathrm{Pt}^{\mathrm{ix}} \\
& \mathrm{Pt}^{\mathrm{iii}}-\mathrm{Cd}-\mathrm{Pt} \\
& \mathrm{Pt}^{\mathrm{viii}}-\mathrm{Cd}-\mathrm{Pt} \\
& \mathrm{Pt}^{\mathrm{ix}}-\mathrm{Cd}-\mathrm{Pt} \\
& \mathrm{Pt}^{\mathrm{iii}}-\mathrm{Cd}-\mathrm{Sr}^{\mathrm{iv}} \\
& \mathrm{P}^{\mathrm{riii}}-\mathrm{Cd}-\mathrm{Sr}^{\mathrm{iv}} \\
& \mathrm{Pt}^{\mathrm{ix}}-\mathrm{Cd}-\mathrm{Sr}^{\mathrm{iv}}
\end{aligned}
$$

120.139 (18)

\begin{tabular}{|c|c|}
\hline $\mathrm{Sr}^{\mathrm{x}}-\mathrm{Cd}-\mathrm{Sr}$ & $113.929(12)$ \\
\hline $\mathrm{Sr}^{\mathrm{xi}}-\mathrm{Cd}-\mathrm{Sr}$ & $66.071(12)$ \\
\hline $\mathrm{Sr}^{\mathrm{vii}}-\mathrm{Cd}-\mathrm{Sr}$ & $113.929(12)$ \\
\hline $\mathrm{Sr}^{\mathrm{viii}}-\mathrm{Cd}-\mathrm{Sr}$ & $79.18(3)$ \\
\hline $\mathrm{Sr}^{\mathrm{rii}}-\mathrm{Cd}-\mathrm{Sr}$ & $100.82(3)$ \\
\hline $\mathrm{Pt}^{\mathrm{iii}}-\mathrm{Cd}-\mathrm{Sr}^{\mathrm{ix}}$ & $121.440(14)$ \\
\hline $\mathrm{Pt}^{\mathrm{viii}}-\mathrm{Cd}-\mathrm{Sr}^{\mathrm{ix}}$ & $58.560(14)$ \\
\hline $\mathrm{Pt}^{\mathrm{ix}}-\mathrm{Cd}-\mathrm{Sr}^{\mathrm{ix}}$ & $58.560(14)$ \\
\hline $\mathrm{Pt}-\mathrm{Cd}-\mathrm{Sr}^{\mathrm{ix}}$ & $121.440(14)$ \\
\hline $\mathrm{Sr}^{\mathrm{iv}}-\mathrm{Cd}-\mathrm{Sr}^{\mathrm{ix}}$ & $113.929(12)$ \\
\hline $\mathrm{Sr}^{\mathrm{x}}-\mathrm{Cd}-\mathrm{Sr}^{\mathrm{ix}}$ & $66.071(12)$ \\
\hline $\mathrm{Sr}^{\mathrm{xi}}-\mathrm{Cd}-\mathrm{Sr}^{\mathrm{ix}}$ & $113.929(12)$ \\
\hline $\mathrm{Sr}^{\mathrm{vii}}-\mathrm{Cd}-\mathrm{Sr}^{\mathrm{ix}}$ & $66.071(12)$ \\
\hline $\mathrm{Sr}^{\mathrm{riii}}-\mathrm{Cd}-\mathrm{Sr}^{\mathrm{ix}}$ & $100.82(3)$ \\
\hline $\mathrm{Sr}^{\mathrm{iii}}-\mathrm{Cd}-\mathrm{Sr}^{\mathrm{ix}}$ & $79.18(3)$ \\
\hline $\mathrm{Sr}-\mathrm{Cd}-\mathrm{Sr}{ }^{\mathrm{ix}}$ & 180.0 \\
\hline $\mathrm{Pt}^{\mathrm{iii}}-\mathrm{Sr}-\mathrm{Pt}$ & $60.28(4)$ \\
\hline $\mathrm{Pt}^{\mathrm{iii}}-\mathrm{Sr}-\mathrm{Pt}^{\mathrm{iv}}$ & $134.76(2)$ \\
\hline $\mathrm{Pt}-\mathrm{Sr}-\mathrm{Pt}^{\mathrm{iv}}$ & $106.214(15)$ \\
\hline $\mathrm{Pt}^{\mathrm{iii}}-\mathrm{Sr}-\mathrm{Pt}^{\mathrm{xii}}$ & $106.214(15)$ \\
\hline $\mathrm{Pt}-\mathrm{Sr}-\mathrm{P}^{\mathrm{xii}}$ & $134.76(2)$ \\
\hline $\mathrm{Pt}^{\mathrm{iv}}-\mathrm{Sr}-\mathrm{Pt}^{\mathrm{xii}}$ & $50.06(3)$ \\
\hline $\mathrm{Pt}^{\mathrm{iii}}-\mathrm{Sr}-\mathrm{Pt}^{\mathrm{xiii}}$ & $106.214(15)$ \\
\hline $\mathrm{Pt}-\mathrm{Sr}-\mathrm{Pt}^{\mathrm{x}} \mathrm{xii}$ & $134.76(2)$ \\
\hline $\mathrm{Pt}^{\mathrm{iv}}-\mathrm{Sr}-\mathrm{Pt}^{\mathrm{xiii}}$ & $110.71(5)$ \\
\hline $\mathrm{Pt}^{\mathrm{xii}}-\mathrm{Sr}-\mathrm{Pt}^{\mathrm{xiii}}$ & $89.75(3)$ \\
\hline $\mathrm{Pt}^{\mathrm{iii}}-\mathrm{Sr}-\mathrm{Pt}^{\mathrm{v}}$ & $134.76(2)$ \\
\hline $\mathrm{Pt}-\mathrm{Sr}-\mathrm{Pt}^{\mathrm{v}}$ & $106.214(15)$ \\
\hline $\mathrm{Pt}^{\mathrm{tiv}}-\mathrm{Sr}-\mathrm{Pt}^{\mathrm{v}}$ & $89.75(3)$ \\
\hline $\mathrm{Pt}^{\mathrm{xii}}-\mathrm{Sr}-\mathrm{Pt}^{\mathrm{v}}$ & $110.71(5)$ \\
\hline $\mathrm{Pt}^{\mathrm{xiii}}-\mathrm{Sr}-\mathrm{Pt}^{\mathrm{v}}$ & $50.06(3)$ \\
\hline $\mathrm{Pt}^{\mathrm{tii}}-\mathrm{Sr}-\mathrm{Cd}^{\mathrm{xiv}}$ & $151.90(4)$ \\
\hline $\mathrm{Pt}-\mathrm{Sr}-\mathrm{Cd}^{\text {xiv }}$ & $91.620(18)$ \\
\hline $\mathrm{Pt}^{\mathrm{iv}}-\mathrm{Sr}-\mathrm{Cd}^{\mathrm{xiv}}$ & $48.779(16)$ \\
\hline $\mathrm{Pt}^{\mathrm{xii}}-\mathrm{Sr}-\mathrm{Cd}^{\mathrm{xiv}}$ & $93.47(3)$ \\
\hline $\mathrm{Pt}^{\mathrm{xiii}}-\mathrm{Sr}-\mathrm{Cd}^{\mathrm{xiv}}$ & $93.47(3)$ \\
\hline $\mathrm{Pt}^{\mathrm{v}}-\mathrm{Sr}-\mathrm{Cd}^{\mathrm{xiv}}$ & $48.779(16)$ \\
\hline $\mathrm{Pt}^{\mathrm{iii}}-\mathrm{Sr}-\mathrm{Cd}^{\mathrm{xiii}}$ & $91.620(19)$ \\
\hline $\mathrm{Pt}-\mathrm{Sr}-\mathrm{Cd}^{\mathrm{xiii}}$ & $151.90(4)$ \\
\hline $\mathrm{Pt}^{\mathrm{iv}}-\mathrm{Sr}-\mathrm{Cd}^{\text {xiii }}$ & $93.47(3)$ \\
\hline $\mathrm{Pt}^{\mathrm{x} \mathrm{xi}}-\mathrm{Sr}-\mathrm{Cd}^{\mathrm{xiii}}$ & $48.779(16)$ \\
\hline $\mathrm{Pt}^{\mathrm{x} \mathrm{iii}}-\mathrm{Sr}-\mathrm{Cd}^{\mathrm{xiii}}$ & $48.779(16)$ \\
\hline $\mathrm{Pt}^{\mathrm{v}}-\mathrm{Sr}-\mathrm{Cd}^{\mathrm{xiii}}$ & 93.47 (3) \\
\hline $\mathrm{Cd}^{\mathrm{xiv}}-\mathrm{Sr}-\mathrm{Cd}^{\mathrm{xiii}}$ & $116.48(4)$ \\
\hline $\mathrm{Pt}^{\mathrm{iii}}-\mathrm{Sr}-\mathrm{Cd}^{\mathrm{ii}}$ & $48.21(2)$ \\
\hline $\mathrm{Pt}-\mathrm{Sr}-\mathrm{Cd}^{\mathrm{ii}}$ & $48.21(2)$ \\
\hline $\mathrm{Pt}^{\mathrm{iv}}-\mathrm{Sr}-\mathrm{Cd}^{\mathrm{ii}}$ & $152.59(2)$ \\
\hline $\mathrm{Pt}^{\mathrm{xii}}-\mathrm{Sr}-\mathrm{Cd}^{\mathrm{ii}}$ & $152.59(2)$ \\
\hline
\end{tabular}

$73.232(13)$

$73.232(13)$

$119.72(4)$

180.0

$54.930(13)$

$54.930(13)$

$59.861(18)$

$59.861(18)$

$64.971(13)$

145.14 (2)

$70.466(13)$

$134.76(2)$

$73.786(15)$

115.029 (13)

$64.971(13)$

$70.466(13)$

$145.14(2)$

$134.76(2)$

$73.786(15)$

115.029 (13)

89.75 (3)

$64.971(13)$

70.466 (13)

$145.14(2)$

$73.786(15)$

$134.76(2)$

115.029 (13)

$129.94(3)$

$69.29(5)$

$64.971(13)$

$145.14(2)$

$70.466(13)$

$73.786(15)$

$134.76(2)$

115.029 (13)

$69.29(5)$

129.94 (3)

89.75 (3)

180.0

109.86 (3)

70.14 (3)

70.14 (3)

109.86 (3)

180.0

119.245 (13)

60.755 (13)

119.245 (13) 


\begin{tabular}{|c|c|c|c|}
\hline $\mathrm{Pt}-\mathrm{Cd}-\mathrm{Sr}^{\mathrm{iv}}$ & $60.755(13)$ & $\mathrm{Pt}^{\mathrm{xii}}-\mathrm{Sr}-\mathrm{Cd}^{\mathrm{ii}}$ & $89.339(16)$ \\
\hline $\mathrm{Pt}^{\mathrm{iii}}-\mathrm{Cd}-\mathrm{Sr}^{\mathrm{x}}$ & $60.755(13)$ & $\mathrm{Pt}^{\mathrm{v}}-\mathrm{Sr}-\mathrm{Cd}^{\mathrm{ii}}$ & $89.339(16)$ \\
\hline $\mathrm{Pt}^{\mathrm{viii}}-\mathrm{Cd}-\mathrm{Sr}^{\mathrm{x}}$ & $119.245(13)$ & $\mathrm{Cd}^{\mathrm{xiv}}-\mathrm{Sr}-\mathrm{Cd}^{\mathrm{ii}}$ & $113.929(12)$ \\
\hline $\mathrm{Pt}^{\mathrm{ix}}-\mathrm{Cd}-\mathrm{Sr}^{\mathrm{x}}$ & $60.755(13)$ & $\mathrm{Cd}^{\mathrm{xiii}}-\mathrm{Sr}-\mathrm{Cd}^{\mathrm{ii}}$ & $113.929(12)$ \\
\hline $\mathrm{Pt}-\mathrm{Cd}-\mathrm{Sr}^{\mathrm{x}}$ & 119.245 (13) & $\mathrm{Pt} \mathrm{t}^{\mathrm{iii}}-\mathrm{Sr}-\mathrm{Cd}$ & $48.21(2)$ \\
\hline $\mathrm{Sr}^{\mathrm{iv}}-\mathrm{Cd}-\mathrm{Sr}^{\mathrm{x}}$ & $180.00(4)$ & $\mathrm{Pt}-\mathrm{Sr}-\mathrm{Cd}$ & $48.21(2)$ \\
\hline $\mathrm{Pt}^{\mathrm{iii}}-\mathrm{Cd}-\mathrm{Sr}^{\mathrm{xi}}$ & $60.755(13)$ & $\mathrm{Pt}^{\mathrm{i} v}-\mathrm{Sr}-\mathrm{Cd}$ & $89.339(16)$ \\
\hline $\mathrm{Pt}^{\mathrm{viii}}-\mathrm{Cd}-\mathrm{Sr}^{\mathrm{xi}}$ & 119.245 (13) & $\mathrm{Pt}^{\mathrm{xii}}-\mathrm{Sr}-\mathrm{Cd}$ & $89.339(16)$ \\
\hline $\mathrm{Pt}^{\mathrm{ix}}-\mathrm{Cd}-\mathrm{Sr}^{\mathrm{xi}}$ & $60.755(13)$ & $\mathrm{Pt}^{\mathrm{xiii}}-\mathrm{Sr}-\mathrm{Cd}$ & $152.59(2)$ \\
\hline $\mathrm{Pt}-\mathrm{Cd}-\mathrm{Sr}^{\mathrm{xi}}$ & $119.245(13)$ & $\mathrm{Pt}^{\mathrm{v}}-\mathrm{Sr}-\mathrm{Cd}$ & $152.59(2)$ \\
\hline $\mathrm{Sr}^{\mathrm{iv}}-\mathrm{Cd}-\mathrm{Sr}^{\mathrm{xi}}$ & $116.48(4)$ & $\mathrm{Cd}^{\mathrm{xiv}}-\mathrm{Sr}-\mathrm{Cd}$ & $113.929(12)$ \\
\hline $\mathrm{Sr}-\mathrm{Cd}-\mathrm{Sr}^{\mathrm{x}}$ & $63.52(4)$ & $\mathrm{Cd}^{\mathrm{xiii}}-\mathrm{Sr}-\mathrm{Cd}$ & $113.929(12)$ \\
\hline $\mathrm{Pt}^{\mathrm{iii}}-\mathrm{Cd}-\mathrm{Sr}^{\mathrm{vii}}$ & 119.245 (13) & $\mathrm{Cd}^{\mathrm{ii}}-\mathrm{Sr}-\mathrm{Cd}$ & $79.18(3)$ \\
\hline $\mathrm{Pt}^{\mathrm{viii}}-\mathrm{Cd}-\mathrm{Sr}^{\mathrm{vii}}$ & $60.755(13)$ & $\mathrm{Pt} \mathrm{t}^{\mathrm{iii}}-\mathrm{Sr}-\mathrm{Sr}^{\mathrm{xv}}$ & $149.861(18)$ \\
\hline $\mathrm{Pt}^{\mathrm{tix}}-\mathrm{Cd}-\mathrm{Sr}^{\mathrm{vii}}$ & $119.245(13)$ & $\mathrm{Pt}-\mathrm{Sr}-\mathrm{Sr}^{\mathrm{xv}}$ & $149.861(18)$ \\
\hline $\mathrm{Pt}-\mathrm{Cd}-\mathrm{Sr}^{\mathrm{vii}}$ & $60.755(13)$ & $\mathrm{Pt}^{\mathrm{iv}}-\mathrm{Sr}-\mathrm{Sr}^{\mathrm{xv}}$ & $55.35(2)$ \\
\hline $\mathrm{Sr}^{\mathrm{iv}}-\mathrm{Cd}-\mathrm{Sr}^{\mathrm{vii}}$ & $63.52(4)$ & $\mathrm{Pt}^{\mathrm{xii}}-\mathrm{Sr}-\mathrm{Sr}^{\mathrm{xv}}$ & $55.35(2)$ \\
\hline $\mathrm{Sr}^{\mathrm{x}}-\mathrm{Cd}-\mathrm{Sr}^{\mathrm{vii}}$ & $116.48(4)$ & $\mathrm{Pt}^{\mathrm{xiii}}-\mathrm{Sr}-\mathrm{Sr}^{\mathrm{xv}}$ & $55.35(2)$ \\
\hline $\mathrm{Sr}^{\mathrm{xi}}-\mathrm{Cd}-\mathrm{Sr}^{\mathrm{vii}}$ & 180.0 & $\mathrm{Pt}^{\mathrm{v}}-\mathrm{Sr}-\mathrm{Sr}^{\mathrm{xv}}$ & $55.35(2)$ \\
\hline $\mathrm{Pt}^{\mathrm{tii}}-\mathrm{Cd}-\mathrm{Sr}^{\text {viii }}$ & $121.440(14)$ & $\mathrm{Cd}^{\mathrm{xiv}}-\mathrm{Sr}-\mathrm{Sr}^{\mathrm{xv}}$ & $58.24(2)$ \\
\hline $\mathrm{Pt}^{\mathrm{v}} \mathrm{iii}-\mathrm{Cd}-\mathrm{Sr}^{\mathrm{viii}}$ & $58.560(14)$ & $\mathrm{Cd}^{\mathrm{xiii}}-\mathrm{Sr}-\mathrm{Sr}^{\mathrm{xv}}$ & $58.24(2)$ \\
\hline $\mathrm{Pt}^{\mathrm{ix}}-\mathrm{Cd}-\mathrm{Sr}^{\text {viii }}$ & $58.560(14)$ & $\mathrm{Cd}^{\mathrm{ii}}-\mathrm{Sr}-\mathrm{Sr}^{\mathrm{xv}}$ & 140.409 (17) \\
\hline $\mathrm{Pt}-\mathrm{Cd}-\mathrm{Sr}^{\mathrm{viii}}$ & $121.440(14)$ & $\mathrm{Cd}-\mathrm{Sr}-\mathrm{Sr}^{\mathrm{xv}}$ & 140.409 (17) \\
\hline $\mathrm{Sr}^{\mathrm{iv}}-\mathrm{Cd}-\mathrm{Sr}^{\text {viii }}$ & $66.071(12)$ & $\mathrm{Pt}^{\mathrm{iii}}-\mathrm{Sr}-\mathrm{Sr}^{\mathrm{xvi}}$ & $53.63(3)$ \\
\hline $\mathrm{Sr}^{\mathrm{x}}-\mathrm{Cd}-\mathrm{Sr}^{\mathrm{viii}}$ & $113.929(12)$ & $\mathrm{Pt}-\mathrm{Sr}-\mathrm{Sr}^{\mathrm{xvi}}$ & $100.38(5)$ \\
\hline $\mathrm{Sr}^{\mathrm{xi}}-\mathrm{Cd}-\mathrm{Sr}^{\mathrm{viii}}$ & $66.071(12)$ & $\mathrm{Pt}^{\mathrm{iv}}-\mathrm{Sr}-\mathrm{Sr}^{\mathrm{xvi}}$ & $151.51(5)$ \\
\hline $\mathrm{Sr}^{\mathrm{vii}}-\mathrm{Cd}-\mathrm{Sr}^{\mathrm{viii}}$ & $113.929(12)$ & $\mathrm{Pt}^{\mathrm{xii}}-\mathrm{Sr}-\mathrm{Sr}^{\mathrm{xvi}}$ & $103.14(3)$ \\
\hline $\mathrm{Pt}^{\mathrm{iii}}-\mathrm{Cd}-\mathrm{Sr}^{\mathrm{iii}}$ & $58.560(14)$ & $\mathrm{Pt}^{\mathrm{xiii}}-\mathrm{Sr}-\mathrm{Sr}^{\mathrm{xvi}}$ & $52.59(2)$ \\
\hline $\mathrm{Pt}^{\mathrm{viii}}-\mathrm{Cd}-\mathrm{Sr}^{\mathrm{iii}}$ & $121.440(14)$ & $\mathrm{Pt}^{\mathrm{v}}-\mathrm{Sr}-\mathrm{Sr}^{\mathrm{xvi}}$ & $92.53(2)$ \\
\hline $\mathrm{Pt}^{\mathrm{ix}}-\mathrm{Cd}-\mathrm{Sr}^{\mathrm{iii}}$ & 121.440 (14) & $\mathrm{Cd}^{\mathrm{xiv}}-\mathrm{Sr}-\mathrm{Sr}^{\mathrm{xvi}}$ & $141.30(3)$ \\
\hline $\mathrm{Pt}-\mathrm{Cd}-\mathrm{Sr}^{\mathrm{iii}}$ & $58.560(14)$ & $\mathrm{Cd}^{\mathrm{xiii}}-\mathrm{Sr}-\mathrm{Sr}^{\mathrm{xvi}}$ & $58.05(2)$ \\
\hline $\mathrm{Sr}^{\mathrm{iv}}-\mathrm{Cd}-\mathrm{Sr}^{\mathrm{iii}}$ & $113.929(12)$ & $\mathrm{Cd}^{\mathrm{ii}}-\mathrm{Sr}-\mathrm{Sr}^{\mathrm{xvi}}$ & $55.88(3)$ \\
\hline $\mathrm{Sr}^{\mathrm{x}}-\mathrm{Cd}-\mathrm{Sr}^{\mathrm{rii}}$ & $66.071(12)$ & $\mathrm{Cd}-\mathrm{Sr}-\mathrm{Sr}^{\mathrm{xvi}}$ & $101.13(5)$ \\
\hline $\mathrm{Sr}^{\mathrm{xi}}-\mathrm{Cd}-\mathrm{Sr}^{\mathrm{rii}}$ & $113.929(12)$ & $\mathrm{Sr}^{\mathrm{xv}}-\mathrm{Sr}-\mathrm{Sr}^{\mathrm{xvi}}$ & $103.81(4)$ \\
\hline $\mathrm{Sr}^{\mathrm{vii}}-\mathrm{Cd}-\mathrm{Sr}^{\mathrm{iii}}$ & $66.071(12)$ & & \\
\hline
\end{tabular}

Symmetry codes: (i) $-x,-y,-z+1$; (ii) $x+1, y, z$; (iii) $-x,-y+1,-z+1$; (iv) $-x-1 / 2,-y+1 / 2,-z+1 / 2$; (v) $-x+1 / 2,-y+1 / 2,-z+1 / 2$; (vi) $x+1 / 2, y-1 / 2, z+1 / 2$; (vii) $x-1 / 2, y-1 / 2, z+1 / 2$; (viii) $x-1, y, z$; (ix) $-x-1,-y+1,-z+1$; (x) $x-1 / 2, y+1 / 2, z+1 / 2$; (xi) $-x-1 / 2,-y+3 / 2,-z+1 / 2$; (xii) $x-1 / 2, y+1 / 2, z-1 / 2$; (xiii) $x+1 / 2, y+1 / 2, z-1 / 2$; (xiv) $x+1 / 2, y-1 / 2, z-1 / 2 ;$ (xv) $-x,-y+1,-z ;$ (xvi) $-x+1 / 2,-y+3 / 2,-z+1 / 2$. 